\title{
Whole grain intake is associated with intakes of other foods and nutrients and some markers of health in the National Diet and Nutrition Survey rolling programme years 1, 2 and 3
}

\author{
K. D. Mann ${ }^{1,2}$, M. S. Pearce ${ }^{2}$, B. McKevith ${ }^{3}$, F. Thielecke ${ }^{4}$ and C. J. Seal ${ }^{1}$ \\ ${ }^{1}$ Institute of Health \& Society, Newcastle University, Newcastle upon Tyne, NE1 4LP, UK, ${ }^{2}$ Human Nutrition \\ Research Centre, Newcastle University, Newcastle upon Tyne NE1 7RU, UK, ${ }^{3}$ Cereal Partners UK, Welwyn Garden \\ City, AL7 1RR, UK and ${ }^{4}$ Cereal Partners Worldwide, Lausanne, Switzerland
}

There is much epidemiological evidence to suggest an inverse association of whole grain consumption with the risk of many noncommunicable diseases. The risk of cardiovascular disease, type 2 diabetes, obesity and some cancers has been shown to be reduced in higher consumers against low or non-consumers of whole grain ${ }^{(1,2,3)}$. We have previously shown that whole grain intake in the National Diet and Nutrition Survey (NDNS) 2008-2011 population is low, with $18 \%$ of adults and $15 \%$ of children/teenagers consuming no whole grain ${ }^{(4)}$. It is important to understand whether the health benefits associated with intake of whole grains are still present at low levels of consumption. The aim of this study was to investigate the association of daily whole grain intake with intakes of other foods and nutrients, and markers of health (anthropometric and blood measures) in the NDNS 2008-2011 population.

The NDNS is a representative dietary survey of households in the UK. Four-day diet diaries were completed by 3073 individuals between 2008 and 2011, along with the collection of anthropometric measures (height, weight, waist/hip ratio and body mass index (BMI)), blood pressure and blood samples. Daily whole grain intake was calculated for each individual, considering foods with any whole grain content, on a dry matter basis. Whole grain intake was also categorised by number of servings, with one serving classified as $16 \mathrm{~g}$ of whole grain, according to the US definition ${ }^{(5)}$.

Over half (57\%) of and children/teenagers (1.5-18 years) and $44 \%$ of adults (19+ years) did not consume one serving of whole grain per day. Around a quarter of adults $(24 \%)$ and children/teenagers $(26 \%)$ consumed one serving of whole grain per day. Significant trends across increasing whole grain servings $(0,1,2,3$ or more) were seen with decreasing C-reactive protein concentrations in adults, decreasing white blood cell count in both adults and children/teenagers and decreasing weight and BMI in children/teenagers. No significant trends were seen with the other blood measures (blood pressure, cholesterol (total, HDH, LDL), triglycerides, creatinine and haemoglobin) and anthropometric measures in adults.

Increased intakes of whole grain was correlated with increasing intakes of milk, yogurt, bread, ready to eat cereal and fruit and vegetable intake. Significantly higher mean intakes of fibre, iron, calcium, potassium, phosphorus, magnesium, thiamin and riboflavin were seen in both adult and child/teenager whole grain consumers compared with non-consumers.

In this population increased whole grain intake was positively associated with white blood cell count, C-reactive protein (in adults only) and anthropometric measures (in children and teenagers only), but not with other markers of health. Whole grain intake was generally low, with over $80 \%$ of adults, teenagers and children not consuming the minimum US recommendation (48 g or 3 servings a day), which may explain the small variation across other markers of health. The nutrient intakes of wholegrain consumers compared with the non-consumers were closer to dietary reference values, particularly for fibre intake, suggesting that increasing whole grain intake is associated with improved diet quality.

1. Cho SS, Qi L, Fahey GC Jr. et al. (2013) Am J Clin Nutr 98, 594-619.

2. Ye EQ, Chacko SA, Chou EL et al. (2012) J Nutr 142, 1304-13.

3. Kyrø C, Skeie G, Loft S et al. (2013) Cancer Causes Control 24, 1363-1374.

4. Mann KD, Pearce MS, McKevith B et al. (2014) abstract submitted to Proc Nutr Soc Summer Meeting.

5. USDA and US. Department of Health and Human Services. (2010) [cited March 2013] Available from: http://www.cnpp.usda.gov/dietaryguide lines.htm. 